\title{
Toward Large-Scale Autonomous Marine Pollution Monitoring
}

\section{Flores, Huber}

2021-03

Flores, H, Hossein Motlagh, N, Zuniga Corrales , A, Liyanage , M , Passananti , M , Tarkoma , S , Youssef , M \& Nurmi , P 2021 , ' Toward Large-Scale Autonomous Marine Pollution Monitoring ' , IEEE Internet of Things Magazine , vol. 4 , no. 1 , pp. 40-45 . https://doi.org/10.1109/IOTM.00

http://hdl.handle.net/10138/329148

https://doi.org/10.1109/IOTM.0011.2000057

acceptedVersion

Downloaded from Helda, University of Helsinki institutional repository.

This is an electronic reprint of the original article.

This reprint may differ from the original in pagination and typographic detail.

Please cite the original version. 


\title{
Toward Large-Scale Autonomous Marine Pollution Monitoring
}

\author{
Huber Flores*, Naser Hossein Motlagh $\dagger$, Agustin Zuniga $\dagger$, Mohan Liyanage*, \\ Monica Passanantił, Sasu Tarkoma†, Moustafa Youssef $\S$, Petteri Nurmi† \\ * Institute of Computer Science, University of Tartu, Estonia \\ $\dagger$ Department of Computer Science, University of Helsinki, Helsinki, Finland \\ $\ddagger$ Department of Chemistry, University of Turin, Italy \\ $\S$ Department of Computer Science and Engineering, American University in Cairo and Alexandria University, Egypt.
}

\begin{abstract}
Marine pollution is a growing worldwide concern, affecting the health of marine ecosystems, human health, and weather patterns. To reduce underwater pollution, it is critical to have access to accurate information about the extent of marine pollutants as otherwise appropriate countermeasures and cleaning measures cannot be chosen. Currently such information is difficult to acquire as existing monitoring solutions are highly laborious or costly, limited to specific pollutants, and have limited spatial and temporal resolution. In this article, we present a research vision of large-scale autonomous marine pollution monitoring that uses coordinated groups of autonomous underwater vehicles (AUV)s to monitor the extent and characteristics of marine pollutants. We highlight key requirements and reference technologies to establish a research roadmap for realizing this vision. We also address the feasibility of our vision, carrying out controlled experiments that address classification of pollutants and collaborative underwater processing, two key research challenges for our vision.
\end{abstract}

Index Terms-AUV; Underwater drones; Marine pollution monitoring; Autonomous robots; Sensing

\section{INTRODUCTION}

Marine pollution is a growing worldwide concern that affects the health of marine ecosystems, weather patterns, and even human health [1]. The main sources of pollution are chemical contaminants and diverse trash, both of which result from human activity. Chemical contaminants result from runoff of chemicals into waterways, with particularly agriculture and sewage being major contributors. Trash, in turn, encompasses manufactured products that end up in marine ecosystems due to littering, winds, lacking waste management or human activity. Regarding trash, plastic debris is particularly problematic as plastics are durable and not subject to biological decomposition. This results in a steady accumulation of plastics, with estimates suggesting that already in 2014 over 5 trillion pieces of plastic were drifting in the oceans [2].

Counteracting problems resulting from marine pollution requires efforts to both prevent pollution entering marine areas and to clean up existing pollutants. Legislative framework, seeking to reduce the use of harmful materials, pursue the former, e.g., through bans or restrictions on single-use plastics [3]. The latter, in turn, requires extensive efforts at mapping the extent of pollutants together with costly and laborious cleaning efforts. Currently, obtaining accurate information about the extent of marine pollutants is difficult as existing measurement solutions are highly laborious or costly, limited to specific pollutants, and have limited spatial and temporal resolution.

In this paper, we envision autonomous marine pollution monitoring as an approach that can improve current state of pollution monitoring and link with existing research activities on autonomous underwater technologies. Our research vision, illustrated in Figure 1, uses autonomous underwater vehicles (AUV)s to identify and classify pollutants in oceans - or other aquatic environments such as lakes and rivers. In our vision, the AUVs can be responsible for monitoring on their own, or they can link with existing infrastructure. Examples of infrastructure include surface stations, such as instrumented buoys moored at anchor locations or vessels that coordinate and collect results from the AUVs, and fixedly deployed underwater infrastructure, such as sensor networks [4] or energy harvesting stations that use marine or seabed activity to generate power [5]. Realising our vision requires new technological solutions in several research fields. For example, targeting appropriate cleaning activity requires sensing techniques that can identify and classify different pollutants. Such techniques need to take into account characteristics of marine environments as the physical and chemical properties of pollutants change when they are exposed to salt, aquatic organisms, UV radiation and other environmental factors [6]. At the same time, sufficient processing power is required to run the sensing models, necessitating new types of collaborative underwater processing infrastructure, highly efficient underwater communication and potentially also new types of energy solutions. To ensure the collected information is of high quality and has sufficient coverage, the AUVs need to coordinate sampling, necessitating advances in underwater localization and AUV coordination. We highlight key research challenges, reflect on current state-of-the-art, and establish a research roadmap for enabling our vision.

We demonstrate the feasibility of our vision by conducting two controlled benchmark experiments that address key research directions of our vision. Our first experiment focuses on underwater pollutant classification using optical (green light) sensing. Optical sensing is a promising candidate for pollutant classification as it has small energy footprint and as it operates robustly in underwater environments. Existing 


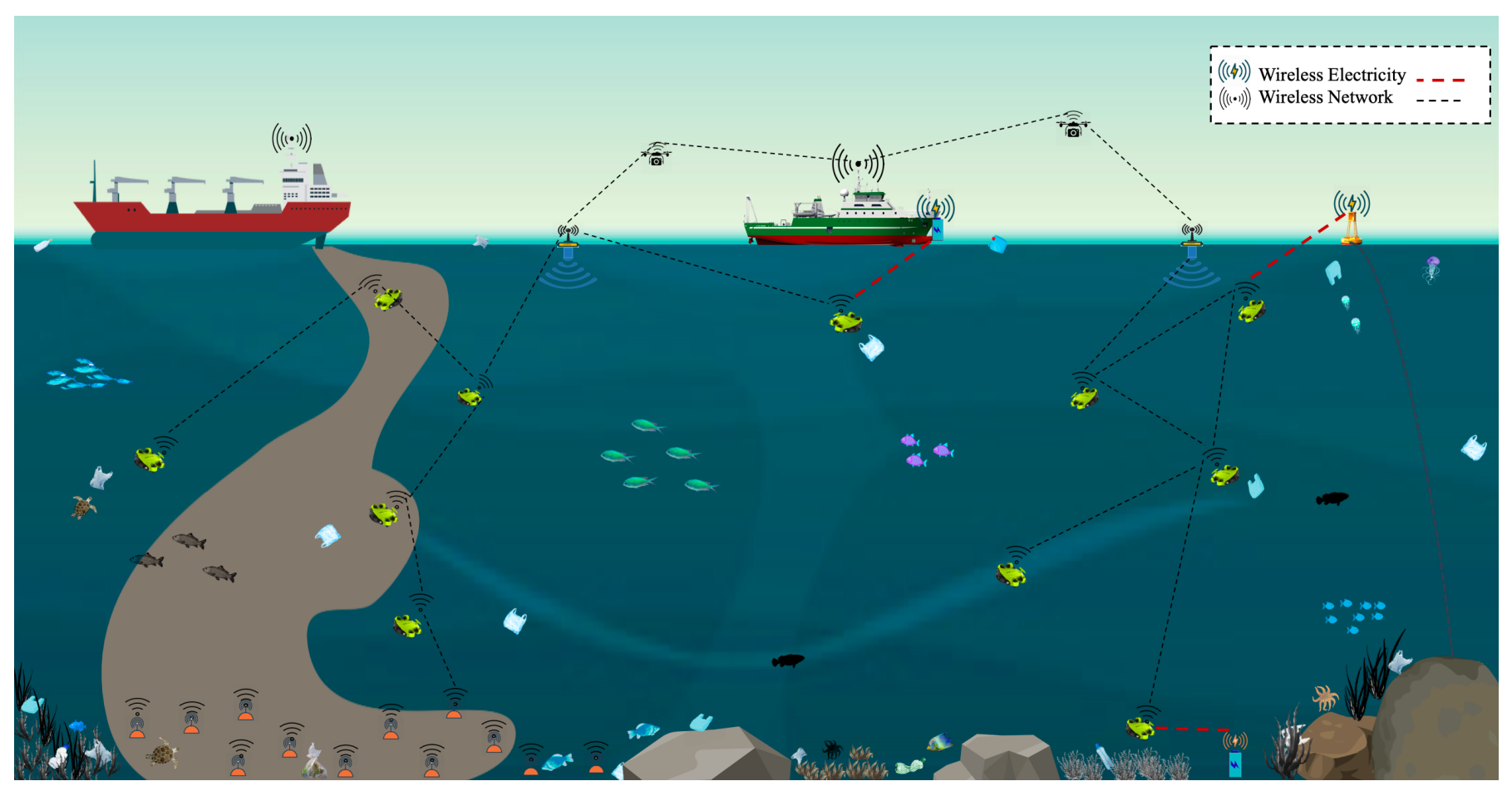

Fig. 1. Vision of AUV deployment for plastic detection underwater.

sensing solutions, such as Fourier transform infrared (FTIR) spectroscopy [7] or computer imaging [8], are poorly suited for underwater sensing due to being sensitive to environmental characteristics. Unlike visible light, infrared has poor propagation characteristics, whereas computer imaging requires heavy processing. Our second experiment focuses on cooperative underwater processing with the aim of supporting debris identification. Due to battery limitations, current AUVs have very limited computational resources making them unsuited for heavy processing. Collaborative processing using underwater micro-clouds can be used to increase complexity of processing without burdening individual AUVs. We analyse the influence of distributed processing underwater, and discuss the implications of our results in underwater contexts.

\section{REQUIREMENTS}

Realising large-scale autonomous marine pollution monitoring requires advances in devices, algorithms, system design, and infrastructure to address limitations of current technology. Next, we discuss key requirements for our vision.

Pollution Detection and Classification: Underwater pollutants come in different shapes and forms, and the optimal countermeasure depends on the pollutant. Indeed, while plastic debris is often highlighted as the main source of pollution, other forms of pollution, such as chemical contaminants (e.g., oil or fertilizer run-off) or microscopic particles resulting from material degradation can be equally hazardous. Interactions between different pollutants also affect pollutant formation, e.g., chemical contaminants can accelerate oxidation, which in turn accelerates plastic degradation resulting in formation of so-called micro-plastics. Determining the most appropriate cleaning operation thus requires capturing detailed information on a wide range of pollutants.

Coordinated Sampling: Covering large underwater areas requires carefully designed sampling strategies and coordination among AUVs. For example, visual surveys rely on transect sampling where information is collected along delineated strips of underwater space. AUVs need to be able to execute transect sampling or related sampling strategies on a largescale to ensure collected information is maximally useful, and can be integrated with existing modelling techniques. As an example, Lamb et al. [9] studied impacts of plastic pollution on reefs through transect samples takes from 159 different reefs over a three year period. At each reef, the surveyed area was between $60-120 \mathrm{~m}^{2}$ and was sampled using three transects which were separated by 20 meters (i.e., $1-3$ grids per delineation). Multiple AUVs working in coordination can achieve this goal, offering much larger coverage and enabling more advanced sampling strategies, such as three-dimensional transects. Pollutants are known to reside at different depths depending on characteristics of the pollutant [1], hence 3D transects and other advanced strategies are essential for capturing the full range of pollutants.

Coordinated Movement and Processing: Besides coordinating sampling areas, AUVs should coordinate their movements to ensure they remain in each other's communication range. Coordinated movement also offers additional benefits, such as the potential for using collaborative sensing or processing strategies for improving the recognition of pollutants. 
Interfaces for Remote Operators: We envision remote operators to be responsible for specifying bounds of areas that need to be monitored and for informing AUVs on potential constraints, e.g., maintaining a minimum distance from sensitive reef vegetation. Within each area, AUVs are then responsible for coordinating the sampling and for performing the monitoring. Supporting the interactions between remote operators and AUVs requires designing interfaces for remote operators and developing systems that can relay the instructions to AUVs operating in the wild.

Fault Tolerance: Ensuring the monitoring captures useful data requires that AUVs can operate for a sufficiently long time (months or even years without human intervention). Beyond energy constraints, this requires high reliability from the AUVs as well as the technologies integrated into them. Malfunctions in the operations of the AUVs can result in the vehicles sinking - potentially making them unrecoverable and part of the pollution problem (AUVs contain metals and plastics). Improving reliability also requires better casing solutions both for the AUVs and the sensors equipped into them. With the exception of highly expensive professional-grade submersibles, smaller-scale AUVs can rarely operate beyond sunlight zone (i.e., below 200 meters).

Pollution Cleaning: Large-scale pollution monitoring requires harnessing thousands or even millions of AUVs to ensure sufficient coverage for the monitoring. Deploying a large number of AUVs is only feasible, if they are sufficiently affordable (less than \$10,000). Current AUVs in this price range are small and have limited capabilities. In terms of cleaning, this means that individual AUVs unlikely can contribute much to cleaning efforts. However, we envision them to take an active role in coordinating surface-based cleaning activities. For example, micro-plastics removal can be operated using specially designed trawlers, whereas larger debris can be collected using surface-based interceptors which can operate using wind and solar power.

\section{Challenges And Enablers}

Enabling fully autonomous marine pollution monitoring is currently difficult due to technological limitations. We next reflect on the current state of technology, and highlight key research challenges in enabling our vision. A summary of the challenges and existing solutions is shown in Table I

Sensing: Accurately detecting and classifying different pollutants requires new types of sensing solutions and systems that can effectively combine sensing modalities. Currently, chemical contaminants can be identified using underwater mass spectrometers [10]. These systems, however, are not suitable for large-scale monitoring as underwater mass spectrometers have limited operating time, are difficult to integrate to submersibles - due to need for a vacuum environment for taking measurements - and suffer from high power draw. Overcoming these challenges requires new power solutions and sensing approaches that can detect the presence of chemical contaminants, and limit the use of spectrometers to areas that are known to be contaminated. For debris, a common approach is to rely on Fourier transform infrared (FTIR) spectroscopy to determine the material of the pollutants [7]. Water heavily absorbs infrared light, making this approach illsuited for underwater use. FTIR also requires special microscopes which are costly, and difficult to integrate with AUVs. Scaling up underwater monitoring thus requires novel lowcost sensing techniques that have low power draw and can operate effectively underwater. Another challenge for debris monitoring is that the materials undergo chemical and physical changes as a result of exposure to UV radiation and aquatic organisms [6]. In practice, it is likely that a combination of sensing techniques needs to be adopted. For example, many AUVs have cameras which can be used to detect the presence of debris $[8]$ and other sensing techniques can then be used to provide a more fine-grained classification (e.g., which type of plastic). Enabling large-scale autonomous pollution monitoring thus requires novel sensing solutions that can operate with low energy footprint, are able to classify different types of pollutants, and work robustly against changes in the physical or chemical composition of materials.

Situational Awareness: Ensuring AUVs coordinate their operations is critical for large-scale autonomous underwater pollution tracking. Autonomy and coordination have been extensively studied in aerial and ground operations [11], but existing techniques cannot be directly adapted to underwater environments. Thus, new solutions and research on adapting existing techniques for underwater environments are needed. AUVs need to have sufficient degree of situational awareness to be able to operate effectively. For example, AUVs need information about obstacles (fish, other aquatic organisms, rocks, and other obstacles). In most aquatic environments, sonar-based solutions are likely sufficient. However, sensitive environments, such as reefs, likely require more precise information. Current high-precision solutions, such as underwater LIDARs, are too expensive, bulky and power consuming for widespread usage, necessitating research on new techniques and miniaturization of existing technologies. There is also a need for effective orchestration mechanisms that allow coordinating AUV operations and interfacing with remote operators.

Underwater Localization: Coordination requires AUVs to be aware of their position relative to other devices. For targeting sampling at the correct areas, it is also critical that AUVs are aware of their global (three dimensional) location. Currently no equivalent of GPS exists for underwater environments with inertial and relative positioning being the best options. Improving coordination and situational awareness thus requires novel underwater localization solutions or hybrid techniques that rely on relative positioning and surface-based stations.

Communication: Coordination between AUVs and other infrastructure is reliant on robust and sufficiently high bandwidth communication links. Underwater communications are among the most widely studied aspects pertaining to our vision with acoustic, optical, ultrasonic, electromagnetic and radio frequency based techniques being examples of solutions 
TABLE I

CURRENT STATE OF AUV TECHNOLOGIES AND KEY CHALLENGES AND RESEARCH TOPICS FOR ENABLING AUTONOMOUS UNDERWATER POLLUTION MONITORING.

\begin{tabular}{|c|c|c|c|}
\hline & State-of-the-Art & Key Research Challenges & Emerging Challenges \\
\hline Sensing & $\begin{array}{l}\text { Algorithms and techniques for } \\
\text { monitoring individual pollution } \\
\text { sources. }\end{array}$ & $\begin{array}{l}\text { Algorithms and techniques for } \\
\text { classifying pollution sources } \\
\text { and detecting multiple pollu- } \\
\text { tants simultaneously. Improved } \\
\text { robustness for variations in un- } \\
\text { derwater conditions. }\end{array}$ & $\begin{array}{l}\text { Approaches to model internal } \\
\text { decomposition and transforma- } \\
\text { tion of materials exposed to en- } \\
\text { vironmental conditions }\end{array}$ \\
\hline $\begin{array}{l}\text { Situational } \\
\text { Awareness }\end{array}$ & $\begin{array}{l}\text { Accurate sonar techniques and } \\
\text { underwater LIDAR technology. } \\
\text { Current LIDAR designs are } \\
\text { bulky, expensive and resource } \\
\text { consuming. }\end{array}$ & $\begin{array}{l}\text { High-resolution and energy- } \\
\text { efficient situational awareness } \\
\text { techniques, including ranging } \\
\text { and camera-based solutions. }\end{array}$ & $\begin{array}{l}\text { Coordinated data collection that } \\
\text { supports scientific sampling, } \\
\text { e.g., belt or grid transects or } 3 \mathrm{D} \\
\text { grid cluster sampling strategies. }\end{array}$ \\
\hline Localization & $\begin{array}{l}\text { Ranging based localization and } \\
\text { underwater dead reckoning } \\
\text { techniques. }\end{array}$ & $\begin{array}{l}\text { Positioning schemes for } 3 \mathrm{D} \text { ab- } \\
\text { solute underwater localization, } \\
\text { improved robustness for relative } \\
\text { positioning. }\end{array}$ & $\begin{array}{l}\text { Hybrid localization solutions } \\
\text { that offer relative and absolute } \\
\text { positioning, e.g., by interacting } \\
\text { with infrastructure residing on } \\
\text { the surface or on the seabed. }\end{array}$ \\
\hline Communications & $\begin{array}{l}\text { Acoustic, optical and electro- } \\
\text { magnetic underwater communi- } \\
\text { cation technologies. }\end{array}$ & $\begin{array}{l}\text { High band short-range commu- } \\
\text { nication technologies and ro- } \\
\text { bust long-range technologies } \\
\text { that can interact with other in- } \\
\text { frastructure }\end{array}$ & $\begin{array}{l}\text { Improving robustness of com- } \\
\text { munication technologies against } \\
\text { water characteristics, such as } \\
\text { currents or other water flows, } \\
\text { temperature, and salinity. }\end{array}$ \\
\hline Design & $\begin{array}{l}\text { Fixed AUV designs that are } \\
\text { tightly sealed and difficult to } \\
\text { expand. }\end{array}$ & $\begin{array}{l}\text { Designs that allow additional } \\
\text { modules, such as sensing or ex- } \\
\text { ternal processing units, to be } \\
\text { integrated into AUV's. }\end{array}$ & $\begin{array}{l}\text { Lightweight casing materials } \\
\text { that offer water and pressure } \\
\text { protection, but do not hamper } \\
\text { sensing or communication func- } \\
\text { tionality. }\end{array}$ \\
\hline $\begin{array}{l}\text { Power and Oper- } \\
\text { ational Time }\end{array}$ & $\begin{array}{l}\text { Battery-based AUV's with short } \\
\text { operating time. Low-efficiency } \\
\text { energy harvesting solutions. }\end{array}$ & $\begin{array}{l}\text { Novel power solutions, includ- } \\
\text { ing wireless charging stations, } \\
\text { underwater energy harvesting } \\
\text { and other solutions. }\end{array}$ & $\begin{array}{l}\text { Collaborative underwater pro- } \\
\text { cessing and offloading for re- } \\
\text { source augmentation and im- } \\
\text { proved energy efficiency. }\end{array}$ \\
\hline
\end{tabular}

that have been proposed [12]. Current technologies, however, are insufficiently mature for unsupervised operation as they suffer from limited range or bandwidth or are sensitive to environmental characteristics such as salinity, water temperature or currents. We envision AUVs to actively share information, e.g., share environmental information or offload camera images for improved debris recognition. This requires communication links with sufficiently high bandwidth. In practice, realising our vision likely requires a combination of long and short-range communication techniques. For example, acoustic communications can be used to connect with surface infrastructure (e.g., instrumented buoys or ships) whereas optical communication offers sufficient range and bandwidth for local connectivity. Optical communication has the added benefit of being able to support simultaneous sensing and communication. Both optical and acoustic communications are sensitive to characteristics of underwater environments and thus there is a need for improving their robustness or for developing alternative communication mediums.
Design: Sensing and computing units traditionally are averse to water, requiring watertight casing when integrated into AUVs. This makes it difficult to extend or adapt AUV operations. Indeed, most AUVs are designed for a dedicated task, such as estimating water velocity [13] or monitoring debris [8]. Realising our vision requires improved AUV designs that are modular and extensible, allowing different types of sensors, additional computing units, and even power sources to be attached to AUVs. At the same time, AUVs need to offer better programming interfaces and SDKs that allow adapting them to different operations. Finally, ensuring sustainability requires research on new types of materials that are durable and harmless to the environment in case accidents happen. As sensing and communication units need encasing, the materials should also be such that they do not degrade sensing or communication performance.

Power and Operational Time: Commercial off-the-shelf AUVs have limited operational time, surviving at most 24 hours between recharges. For example, PowerRay, one of the 

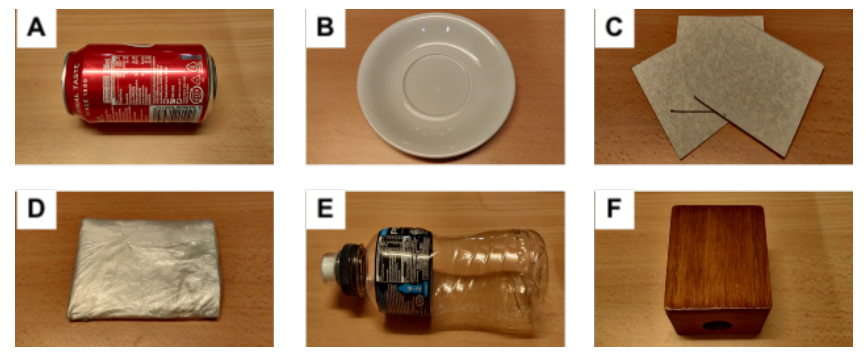

Fig. 2. Debris materials. A. Aluminium can, B. Ceramic plate, C. Paperboard, D. Plastic bag, E. Plastic bottle, F. Wooden toy.

most popular commercial underwater drones, reportedly has a four hour operational time while submerged. To avoid loss, the internal processing units of AUVs commonly integrate routines that estimate travel times and prevent travel if there is not enough power for a return trip. Large-scale monitoring thus requires designated station points where AUVs can be moored and charged. Optimally charging would happen without human intervention, e.g., using buoys that harvest solar energy or seabed sensors harvesting energy from currents and seabed motions [5]. This is difficult to achieve as station points require additional deployment and maintenance. Besides novel power solutions, operational time can be improved by designing mechanisms for energy-efficient operations. For example, image processing operations can be performed collaboratively or offloaded to surface-based infrastructure. Indeed, in the near future it may be possible for AUVs to link with data centers that are located on the surface [14].

\section{FEASIBILITY EXPERIMENTS}

We address feasibility of our vision through two benchmark experiments addressing two of the key research challenges described in the previous section. Our first experiment targets energy-efficient classification of debris based pollutants using optical green light sensing, whereas our second experiment examines the feasibility of using commercial-off-the-shelf devices for augmenting the processing resources of AUVs. We next briefly summarize the experimental setup of our two benchmarks. In the experiments we rely on measurements from controlled testbeds to have better control over experimental variables. We have also separately verified the feasibility of these technologies in AUV operations by integrating the sensors onto a PowerRay underwater drone.

\section{A. Underwater Material Sensing}

Having a complete view of pollutant types and characteristics is necessary for targeting appropriate cleaning actions and estimating the severity of the current pollution situation. Current monitoring solutions are unable to achieve this as they can only detect the presence of pollutants without being able to classify them. Our first experiment demonstrates that optical sensors available on commercial-off-the-shelf devices can be used to perform coarse-grained debris classification. We focus

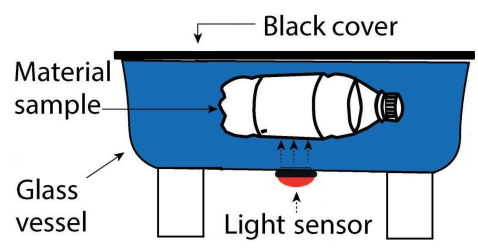

(a) Underwater material sensing setup.

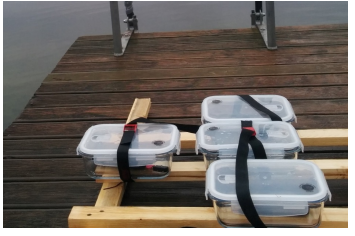

(b) Micro-cloud deployment.
Fig. 3. Our controlled testbed for underwater material sensing and collaborative processing.

on optical sensing as the sensors are inexpensive, have low power draw, and are capable of operating normally underwater.

Apparatus: We perform our experiment using a commercial off-the-shelf smartwatch (Samsung Gear S3 Frontier) which integrates two green light LED lights and a photo-receptor. We focus on green light due to its short wavelength, which makes it excellent at penetrating water.

Materials: We test against common everyday objects with different materials. The objects are shown in Figure 2 and they were chosen as representative examples of common types of underwater debris. The objects are: a snack box (paperboard, PAP21), a plastic bag (high-density polyethylene HDPE), a plastic bottle (polyethylene terephthalate PET), a soft drink can (aluminium, ALU 41), a small ceramic plate (feldspar) and a wooden toy box (solid walnut oak).

Setup: We place each object in turn into a glass container covered with a non-reflective (black) lid. The smartwatch is taped outside the container, directly below the measured object; see Figure 3(a) For each object, we perform 4 sets of measurements with each set consisting of 6 repetitions. Each repetition contains light intensity measurements sampled with $100 \mathrm{~Hz}$ frequency over a 90 second period. The four sets were divided into a $2 \times 2$ design with sensing medium (air cf. water) and luminosity (ambient cf. darkness) as the experimental conditions. The two conditions for sensing medium refer to whether the container was empty or filled with water. The former emulates having a water-proof casing on the surface, whereas the latter emulates detection in an underwater environment. The luminosity conditions correspond to a case where the container is unobstructed, emulating case where ambient light seeps into water, and darkness, emulating direct contact between the sensor and the object. The average strength of ambient light was measured as 15.51x. Darkness was achieved by covering the container with a cardboard box. We separately verified that luminosity inside the box was zero.

\section{B. Underwater Distributed Processing}

Scaling up autonomous pollution monitoring requires participating AUVs to be affordable yet sufficiently powerful to carry out the required operations. Currently this is not the case, with affordable AUVs having limited computational power and a short operational time. For example, most AUVs integrate a Raspberry PI (1.3Ghz CPU) or equivalent micro-controller, and have a maximum operational time of four hours without recharging. In our second experiment, we demonstrate how 
the processing capability can be augmented by integrating additional computing capability, in the form of a simplistic micro-cloud, with the AUV.

Apparatus: We emulate an extendable AUV design and assume a micro-cloud consisting of devices providing additional computing power is attached onto the surface of the AUV. In the experiment, we construct the micro-cloud from four LG Nexus 5 smartphones. Each smartphone is placed inside a sealed glass container. To control for communication distance, instead of using the AUV, we attach the glass containers on a wooden structure equidistant from each other; see Figure 3(b). During the experiment we submerge the casings to assess underwater performance.

Task: As the experimental task we consider object recognition from a video-feed. We chose this task as it emulates the computational needs of vision-based underwater debris recognition. As the video feed, we consider a set of 50 images $(224 \times 224$ resolution) taken from ImageNet. For recognition, we use a pre-trained and quantized mobilenet model (_v1_1.0_224) which is deployed on each smartphone participating in the micro-cloud.

Prototype: We implemented a proof-of-concept Android prototype that runs on the smartphones. The app uses $\mathrm{WiFi}$ to connect to the other participating devices. One device is randomly chosen as a master that initiates computing on the other devices, which act as workers. The master device receives a sequential video feed and sends individual frames to the worker devices in a round robin fashion. Once a worker finishes its task, it sends the results back to the master.

Metrics: As the evaluation metrics we consider task completion rate, i.e., the number of frames processed by slaves, and task success rate, i.e., the number of returned frames that are successfully received by master. As our focus is on assessing feasibility of underwater offloading and as we use a pre-trained model, evaluating object recognition performance was not meaningful and is omitted. The benefits of running object recognition on smart device micro-clouds have been demonstrated in our previous work [15] and hence we focus on task completion and success rates.

\section{RESUlts}

\section{A. Underwater Material Sensing}

We first use statistical significance testing to verify that the green light intensity measurements of different materials indeed have sufficient variation to support debris identification. Kruskal-Wallis test showed significant differences for all conditions: air - ambient $\left(\chi^{2}=6900, \eta^{2}=0.95, \mathrm{p}<.001\right)$, water - ambient $\left(\chi^{2}=7003, \eta^{2}=0.97, \mathrm{p}<.001\right)$, air - darkness $\left(\chi^{2}=7005, \eta^{2}=0.97, \mathrm{p}<.001\right)$ and water - darkness $\left(\chi^{2}=7004, \eta^{2}=0.97, \mathrm{p}<.001\right)$. Here air and water refer to the conditions for sensing medium, i.e., having the glass container empty or filled with water to emulate underwater environment, whereas ambient and darkness refer to the two luminosity conditions; see Section IV-A. Posthoc comparisons
TABLE II

CLASSIFICATION ACCURACY IN DIFFERENT EXPERIMENTAL CONDITIONS.

\begin{tabular}{lccc}
\hline Cross Validation test & k-NN & Random forest & Average \\
\hline All conditions 6-folds & 70.8 & 72.9 & $\mathbf{7 1 . 9}$ \\
Ambient 6-folds & 81.2 & 82.2 & $\mathbf{8 1 . 7}$ \\
Darkness 6-folds & 81.2 & 77.1 & $\mathbf{7 9 . 2}$ \\
Air 6-folds & 76.0 & 71.9 & $\mathbf{7 4 . 0}$ \\
Underwater 6-folds & 66.7 & 64.6 & $\mathbf{7 2 . 5}$ \\
\hline Average & $\mathbf{7 4 . 8}$ & $\mathbf{7 3 . 5}$ & $\mathbf{7 4 . 1}$ \\
\hline
\end{tabular}

(Dunn-Bonferroni) verified that the differences for objects also were statistically significant in all experiment conditions.

We next demonstrate that optical sensing can provide a coarse-grained classification of debris by using the light intensity measurements to run classification experiments. We test using two simple classifiers, a random forest model and a $k$-nearest neighbour classifier, and under different evaluation scenarios. We focus on simple classifiers as any models deployed on AUVs need to be simple to ensure they are as energy-efficient as possible. The results of our experiments are shown in Table II. We separately tested how a change in sensing medium (air cf. water) or luminosity (darkness cf. ambient) affects performance. When all four test conditions are included, the classification accuracy is around $70 \%$. The best classification performance, slightly over $80 \%$, is obtained when both testing and training measurements are from environments with similar luminosity values (rows ambient and darkness in the table). Changes in sensing medium have some effect on performance, but these are not as pronounced as in the case of luminosity changes. Overall, the results demonstrate that optical sensing can be used to provide a coarse-grained classification of different pollutants, as long as the training samples provided by the machine learning algorithms are sufficiently similar to the environment where the AUVs operate.

\section{B. Underwater Distributed Processing}

We first consider the processing time of the micro-cloud is affected by the number of devices participating in it. Results of this evaluation are shown in Figure 4. As expected, including more workers decreases processing time. Even with one worker device, it is possible to reach a $30 \mathrm{fps}$ processing frequency, demonstrating that underwater micro-clouds could be used to support tasks that rely on real-time analysis of camera images. Increasing the number of workers would allow using faster rate of video or higher image resolution. We also assessed how encasing or submerging the devices affects their computing performance. From the figure we observe these to have a small but overall negligible effect (approximately $5 \mathrm{~ms}$ for the encasing and approximately $20 \mathrm{~ms}$ for submerging).

Next, we evaluate task success rate by measuring the percentage of frames that the workers return to the master, i.e., loss due to communication failures. When the devices operate above the surface, the success rate is $100 \%$ - also when the devices are encased in the glass container. Once the 


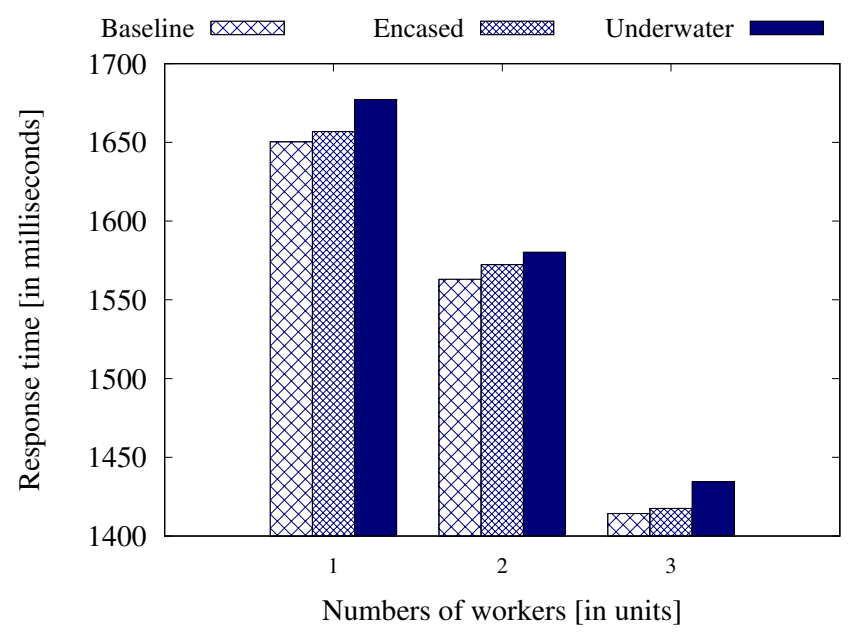

Fig. 4. Collaborative processing with waterproof micro-clouds.

devices are submerged, the success rate decreases - as could be expected. When devices are close to each other (within $7 \mathrm{~cm}$ ), success rate remains at $100 \%$. However, once distance exceeds this, the performance drops, first to $70 \%$ at around $10 \mathrm{~cm}$ distance, and then to $0 \%$ beyond $10 \mathrm{~cm}$ distance. Our results thus suggest that underwater collaborative processing itself is feasible with reasonably inexpensive components, but there is a need for short-range communications solutions that can operate efficiently underwater. Alternatively, devices could operate inside a single container, in which case wireless communications would be sufficient. However, in this case, the weight of the container and heat accumulation inside it easily become an issue for operating the AUV.

\section{DISCUSSION}

Thus far we have focused on technological challenges in enabling large-scale autonomous marine pollution monitoring. In practice, there are many other challenges also, e.g., related to the operations and the overall service ecosystem. Below we briefly highlight some of these aspects:

Marine Hazards: AUV technology operating in aquatic ecosystems can be perceived as invasive by wildlife and be subjected to attacks or other unpredictable behaviors. These operations may be harmful to aquatic wildlife or result in damage to AUVs. Overcoming this issue requires better understanding of different AUV designs and how they are perceived by different species, and algorithms that allow AUVs to adapt their behavior to minimize disruptions to aquatic wildlife.

Further Issues: We have sought to highlight key research challenges related to technology and to demonstrate the feasibility of underwater pollution monitoring. In practice, several other factors affect the adoption of AUVs. For instance, operational regulations and legislation on operating AUVs, especially in marine areas intersecting borders of multiple countries. Other factors include integration of AUVs with legacy technologies, such as already deployed underwater sensor networks, and fabrication of suitable materials for manufacturing of AUVs, e.g., using 3D printing.
Marine Data: Our results demonstrated how combinations of different existing technologies could be adopted for improving resolution of underwater pollution monitoring. In particular, collaborative underwater processing supports applications that require processing and analysis of camera images whereas optical sensing could be used to support more fine-grained classification into different materials. Improving the maturity of these technologies, and supporting the development of new sensing modalities, would benefit from large-scale datasets that can be used to train and test different solutions.

Value Added Services and Stakeholders: Sustainability of marine life and water resources is a primary concern for governmental institutions across the globe, and aquaculture industries in open waters. Thus, these are primary entities that will foster large-scale and permanent deployments of AUVs. Since AUV deployment is relatively easy and flexible, other groups can also benefit indirectly from spontaneous deployments to monitor emergency events underwater and to offer other monitoring services, e.g., oil spills or fishery monitoring. Supporting the requirements of external stakeholders requires new types of service orchestration schemes that allow easily adapting AUVs for different monitoring tasks, data visualizations that can provide actionable feedback on detection results, and even privacy and security solutions that help preserve data confidentiality in case of unauthorized access, e.g., due to previous loss of equipment.

\section{SUMmARY AND CONCLUSIONS}

We developed a vision of large-scale autonomous marine pollution monitoring that delivers accurate information about the extent and characteristics of pollutants at high spatial and temporal resolution. By contrasting our vision against current deployments, we identified key research challenges and discussed relevant reference technologies that can support delivering on our vision. These challenges include development of new sensing techniques that can operate under naturalistic degradation patterns, coordination techniques for AUVs, and underwater positioning and networking solutions that improve the process that is used for collecting measurements. Besides highlighting the key challenges and technologies, we demonstrated the feasibility of our vision through small-scale controlled experiments that addressed distributed and cooperative processing, and pollutant detection and classification, two key research challenges for our vision.

\section{ACKNOWLEDGEMENTS}

This research is supported by the European Regional Funds through the IT Academy Programme, Academy of Finland grant 317875 and Jorma Ollila grant 201920082.

\section{REFERENCES}

[1] F. Thevenon, C. Carroll, and J. Sousa (Eds.), "Plastic debris in the ocean: the characterization of marine plastics and their environmental impacts," International Union for Conservation of Nature (IUCN), situation analysis report, 2015. 
[2] M. Eriksen, L. C. Lebreton, H. S. Carson, M. Thiel, C. J. Moore, J. C. Borerro, F. Galgani, P. G. Ryan, and J. Reisser, "Plastic pollution in the world's oceans: more than 5 trillion plastic pieces weighing over 250,000 tons afloat at sea," PloS one, vol. 9, no. 12, p. e111913, 2014

[3] R. E. Schnurr, V. Alboiu, M. Chaudhary, R. A. Corbett, M. E. Quanz, K. Sankar, H. S. Srain, V. Thavarajah, D. Xanthos, and T. R. Walker, "Reducing marine pollution from single-use plastics (sups): A review," Marine Pollution Bulletin, vol. 137, pp. 157 - 171, 2018.

[4] J. Heidemann, M. Stojanovic, and M. Zorzi, "Underwater sensor networks: applications, advances and challenges," Philosophical Transactions of the Royal Society A: Mathematical, Physical and Engineering Sciences, vol. 370, no. 1958, pp. 158-175, 2012.

[5] H. F. Rezaei, A. Kruger, and C. Just, "An energy harvesting scheme for underwater sensor applications," in 2012 IEEE International Conference on Electro/Information Technology. IEEE, 2012, pp. 1-4.

[6] M. Passananti, F. Temussi, M. R. Iesce, L. Previtera, G. Mailhot, D. Vione, and M. Brigante, "Photoenhanced transformation of nicotine in aquatic environments: Involvement of naturally occurring radical sources," water research, vol. 55, pp. 106-114, 2014.

[7] F. Huth, M. Schnell, J. Wittborn, N. Ocelic, and R. Hillenbrand, "Infrared-spectroscopic nanoimaging with a thermal source," Nature materials, vol. 10, no. 5, pp. 352-356, 2011.

[8] Y. Wang, D. Wang, Q. Lu, D. Luo, and W. Fang, "Aquatic debris detection using embedded camera sensors," Sensors, vol. 15, no. 2, pp. 3116-3137, 2015.

[9] J. B. Lamb, B. L. Willis, E. A. Fiorenza, C. S. Couch, R. Howard, D. N. Rader, J. D. True, L. A. Kelly, A. Ahmad, J. Jompa et al., "Plastic waste associated with disease on coral reefs," Science, vol. 359, no. 6374, pp. 460-462, 2018.

[10] J. A. Farrell, S. Pang, and W. Li, "Chemical plume tracing via an autonomous underwater vehicle," IEEE Journal of Oceanic Engineering, vol. 30, no. 2, pp. 428-442, 2005.

[11] N. H. Motlagh, T. Taleb, and O. Arouk, "Low-altitude unmanned aerial vehicles-based internet of things services: Comprehensive survey and future perspectives," IEEE Internet of Things Journal, vol. 3, no. 6, pp. 899-922, 2016.

[12] X. Che, I. Wells, G. Dickers, P. Kear, and X. Gong, "Re-evaluation of rf electromagnetic communication in underwater sensor networks," IEEE Communications Magazine, vol. 48, no. 12, pp. 143-151, 2010.

[13] C. C. Eriksen, T. J. Osse, R. D. Light, T. Wen, T. W. Lehman, P. L. Sabin, J. W. Ballard, and A. M. Chiodi, "Seaglider: A longrange autonomous underwater vehicle for oceanographic research," IEEE Journal of oceanic Engineering, vol. 26, no. 4, pp. 424-436, 2001.

[14] B. Cutler, S. Fowers, J. Kramer, and E. Peterson, "Dunking the data center,' IEEE Spectrum, vol. 54, no. 3, pp. 26-31, 2017.

[15] E. Lagerspetz, X. Li, J. Hamberg, H. Flores, P. Nurmi, N. Davis, and S. Helal, "Pervasive data science on the edge," IEEE Pervasive Computing, 2019.

\section{BIOGRAPHIES}

Huber Flores: is an Associate Professor at the University of Tartu. He completed his Ph.D. in Computer Science, University of Tartu, Estonia in 2015. His research interest include distributed, mobile and pervasive computing systems. Contact him at huber.flores@ut.ee

Naser Hossein Motlagh: is a Postdoctoral Researcher at the Department of Computer Science, University of
Helsinki. He completed his D.Sc. in Networking Technology, Aalto University, Finland in 2018. His research interests include IoT, WSN, UAVs and AUVs. Contact him at naser.motlagh@helsinki.fi

Agustin Zuniga: is a Doctoral candidate at the Department of Computer Science, University of Helsinki. His current research focuses on collaborative mobile sensing and pervasive data science. Contact him at agustin.zuniga@helsinki.fi

Mohan Liyanage: is a Postdoctoral researcher at the University of Tartu, Estonia. He completed his Ph.D. in Computer Science, University of Tartu, Estonia in 2018. His research interests include, mobile edge computing, Internet of Things and pervasive systems. Contact him at mohan.liyanage@ut.ee

Monica Passananti: is an Assistant Professor at the Department of Chemistry, University of Turin, Italy. She completed her Ph.D. in Chemical Sciences, University of Naples Federico II, Italy in 2013. Her research interest include environmental processes and pollutants degradation in the environment. Contact her at monica.passananti@unito.it

Sasu Tarkoma: is a Full Professor at the Department of Computer Science, University of Helsinki. He completed his Ph.D. in Computer Science at the University of Helsinki in 2006. His research interests include mobile computing, Internet technologies, and AI. Contact him at sasu.tarkoma@helsinki.fi

Moustafa Youssef: is a Professor of computer and systems engineering at American University in Cairo and Alexandria University, Egypt. He completed his Ph.D. in Computer Science, University of Maryland in 2004. His research interests include mobile wireless networks, mobile computing, location determination technologies, pervasive computing, and network security. Contact him at moustafa@alexu.edu.eg

Petteri Nurmi: is an Associate Professor at the University of Helsinki. He completed his Ph.D. in Computer Science at the University of Helsinki in 2009. His research interests include distributed systems, pervasive data science, and sensing systems. Contact him at petteri.nurmi@helsinki.fi 